\title{
e-Phaïstos
}

e-Phaïstos

Revue d'histoire des techniques / Journal of the history

of technology

V-2 | 2016

Histoire des techniques en Afrique de l'Ouest

\section{La Maison de Salomon : contribution à l'histoire du patronage scientifique et technique. France et Angleterre, ca. 1600 - ca. 1660}

Solomon's House : Contribution to the History of Scientific and Technical

Patronage, France and England (ca. 1600-ca. 1660)

\section{Aurélien Ruellet}

\section{OpenEdition}

Journals

Édition électronique

URL : http://journals.openedition.org/ephaistos/6018

DOI : 10.4000/ephaistos.6018

ISSN : 2552-0741

Éditeur

IHMC - Institut d'histoire moderne et contemporaine (UMR 8066)

Édition imprimée

Date de publication : 15 juin 2016

Pagination : $110-15$

ISSN : 2262-7340

Référence électronique

Aurélien Ruellet, « La Maison de Salomon : contribution à l'histoire du patronage scientifique et technique. France et Angleterre, ca. 1600 - ca. 1660 », e-Phaïstos [En ligne], V-2 | 2016, mis en ligne le 15 mars 2020, consulté le 15 septembre 2020. URL : http://journals.openedition.org/ephaistos/6018 


\title{
La maison de Salomon : contribution à I'histoire du patronage scientifique et technique. France et Angleterre, ca. 1600-ca.1660
}

\section{Thèse ayant obtenu le prix de thèse de la Société Française d'Histoire des Sciences et des Techniques 2015}

\author{
Thèse de doctorat d'histoire \\ Université François Rabelais de Tours \\ Centre d'Etudes Supérieures de la Renaissance (CESR) \\ Soutenue le $1^{\mathrm{er}}$ mars 2014 \\ Un volume (417 p.) - un volume d'annexes (278 p.)
}

Directeurs de thèse :

Pascal BRIOIST, professeur à l'université François Rabelais de Tours

Jury:

Gérald CHAIX, professeur, université François Rabelais de Tours (président du jury)

Mark GREENGRASS, professeur, université de Sheffield, Royaume-uni

Liliane HILAIRE-PEREZ, professeure, université Paris 7 Diderot (rapporteure)

Stephen PUMFREY, senior lecturer, université de Lancaster, Royaume-Uni

François-Joseph RUGGIU, professeur, université Paris 4 Sorbonne
Monsieur le président,

Madame et Messieurs les Membres du Jury,

La thèse emprunte son titre à La Nouvelle Atlantide de Francis Bacon. L'un des passages les plus fameux de cette utopie publiée en 1627 est la description, sur l'île de Bensalem qu'abordent les navigateurs, de la «maison de Salomon", une institution dédiée à l'avancement des connaissances théoriques et pratiques. Le gouverneur de cette maison dépeint aux visiteurs un fonctionnement collaboratif et hiérarchisé où le partage des tâches permet de recenser, conserver et améliorer les savoir-faire techniques et les inventions. Francis Bacon créait là un modèle puissant à la postérité conséquente. Il est en effet devenu banal dans l'historiographie de situer dans cet établissement idéal l'origine des académies et institutions scientifiques qui fleurissent dans la seconde moitié du siècle ${ }^{1}$.

Le parti-pris initial de ce travail est d'aborder la protection des savoirs scientifiques et techniques en 
focalisant l'observation sur les «institutions sans murs », avant que les institutions formelles dotées de règlements et de personnels n'apparaissent. Il s'agit donc d'établir une géographie des sites institutionnels et sociaux à partis desquels les savoirs scientifiques et techniques et ceux qui les portent peuvent être protégés, encouragés ou rétribués. L'enquête porte sur les royaumes anglais et français entre le début du XVII ${ }^{e}$ siècle et la décennie 1660 qui voit l'apparition des académies scientifiques.

La notion de patronage est mobilisée dans ce travail pour désigner ces formes variées de protection. Elle est ici préférée au concept de mécénat. Celui-ci est pourtant prisé par l'historiographie et les commentateurs de langue française, mais il tend à euphémiser les rapports de domination et la dimension économique des relations qui unissent protecteur et protégé. De plus, le mécénat ne peut être réservé qu'aux cas les plus spectaculaires de protection culturelle et semble peu apte à rendre compte, par exemple, des médiations administratives. Pour ces raisons, la thèse privilégie la notion de patronage qui a fait l'objet de forts développements en histoire sociale lors des dernières décennies et qui a le mérite, à l'instar du terme anglais patronage, de garder mêlés différents aspects de la protection que le français sépare. Par patronage, et sans prétendre contribuer à une forte conceptualisation du phénomène, on inclut ici une grande variété d'actions : le mécénat culturel; la commensalité et la domesticité ; le patronage administratif; les investissements. Ce patronage est dit par commodité scientifique ou technique, lorsqu'il a pour but ou pour effet de favoriser les menées ou les carrières d'individus se livrant à des activités scientifiques ou techniques, quand bien même la protection ne porte pas directement sur ce type d'occupation. Ce faisant, nous souhaitions désenclaver l'abord du patronage scientifique et technique, qu'on ne peut séparer qu'artificiellement des autres formes de soutien que connaissent les sociétés de l'époque moderne.
L'étude porte prioritairement sur les individus usant des savoirs mathématiques, entendu au sens large qui est celui des acteurs du temps : mathématiques pures, mais aussi « mathématiques mixtes » pour reprendre un vocable baconien. Pour aborder cette institution sans mur, l'enquête a mobilisé des sources diverses, en Angleterre comme en France : sources gouvernementales, archives des cours de justice, ouvrages scientifiques du temps, correspondances, actes notariés pour la France.

Le patronage aristocratique grandiose, fait de pensions et d'emplois dans les maisons des Grands, s'il est souvent convoité et immanquablement célébré par les dédicaces, semble être une ressource rare. La première partie revient sur ce problème à travers deux chapitres. Le premier est consacré aux différentes formes du mécénat. Les degrés de la dépendance sont d'abord rappelés, de la commensalité conviviale - le gîte et le couvert offerts - à la domesticité parfois humiliante, que cherchent à éviter dans la mesure du possible ceux qui aspirent à vivre sans maître. Les emplois de secrétaires, de bibliothécaires, de chapelains, de précepteurs ou médecins personnels sont au demeurant plutôt rares. Quant au mécénat à l'état pur, la protection sans l'accomplissement de services extrascientifiques, il semble n'avoir existé que pour les plus grandes fortunes aristocratiques. La protection des cénacles savants est une autre tentation aristocratique, mais elle achoppe souvent sur la difficile conciliation des intérêts savants et des intérêts des Grands. Le cas de l'académie Montmor qui se réunit à Paris au cours des années 1650 et 1660 l'illustre bien. L'autorité de l'hôte ne suffit pas à éteindre les disputes parfois acrimonieuses et la présence d'un parterre aristocratique tend à avilir les pratiques scientifiques en les déplaçant vers le registre du spectaculaire et du divertissant. L'exemple des observations d'éclipses de Lune et de Soleil montre que l'activité savante ne bénéficie pas de la proximité des Grands : les compilations imprimées permettent d'établir que ces observations se 
déroulaient le plus souvent hors de toute assistance aristocratique. Lorsque celle-ci était présente, les savants avaient davantage pour mission de divertir et de flatter le prince que d'effectuer de vraies mesures astronomiques. Les savants en viennent à désespérer du soutien des Grands qui se montrent réticents à « dépenser un double pour l'avancement des sciences" pour reprendre une formule du virtuoso Jacques-Alexandre Le Tenneur ${ }^{2}$. Dans ce contexte, l'appel à Colbert et à l'Etat pour protéger les cercles savants et émuler la société royale anglaise sonne comme un constat désabusé de la faillite du mécénat aristocratique.

Le deuxième chapitre aborde cette question par le biais des dédicaces des ouvrages scientifiques, dont on questionne d'abord l'intérêt pour l'histoire sociale. La coutume veut que l'auteur d'un ouvrage, en remerciement d'une faveur ou pour en obtenir une, l'offre à une personne de qualité en accompagnant le don matériel d'une lettre dédicatoire en tête du volume. L'omniprésence des dédicaces dans la production imprimée du temps a tôt fait de discréditer ce type de texte : ressassant ad nauseam les mêmes tropes et les mêmes flatteries, les dédicaces semblent d'abord obéir aux modèles épistolaires des secrétaires et des traités de civilité. De fait, les dédicaces sont des écrits courtisans qui révèlent en creux, par les formes d'adresse, la hiérarchie et la distance sociales. Leur rôle rhétorique comme dispositif paratextuel a également été bien étudié : les dédicaces, comme les préfaces ou les épîtres au lecteur, sont un dispositif de captatio benevolentiae dessinant la figure du lecteur généreux. Ces traits communs semblent disqualifier la dédicace comme source d'histoire sociale. Toutefois, ces textes trahissent parfois de vraies relations de protection. Outre-manche, l'astrologue royaliste George Wharton, lors des années de l'Interrègne, tend ainsi à dédicacer ses almanachs à ses voisins " cavaliers » du Berkshire, signalant par là les dettes qu'il avait contractées à l'égard de protecteurs. Le cas d'un praticien mathématique peu connu montre par ailleurs que les dédicaces ne pointent pas toutes vers des liens de type mécénique. Didier Henrion, compilateur didactique, vulgarisateur et introducteur de mathématiques étrangères, a dédicacé la quasitotalité de ses nombreuses publications, pour l'essentiel aux élèves qui constituent la clientèle de ses cours «en chambre » ou à leur famille. La dédicace sert ici de publicité implicite sur le marché de l'éducation nobiliaire. Mais pour Henrion, l'imprimé est le lieu d'une autre forme de patronage : le praticien mathématique bénéficie en effet de la protection d'un secrétaire du roi, Michel Renouard, qui lui octroie des privilèges d'impression assortis de conditions avantageuses. Les termes de ces privilèges permettent à Henrion d'assoir la rentabilité de son entreprise éditoriale et de négocier en position de force avec les librairesimprimeurs. Lorsque Henrion adresse une dédicace à Renouard en tête de l'une de ses publications, ce n'est donc pas tant pour le remercier d'une éventuelle gratification pécuniaire, que pour avoir favorisé son insertion sur le marché du livre mathématique. Au terme de la première partie, le faux antagonisme entre patronage et marché est donc critiqué.

La deuxième partie entreprend d'aborder les logiques étatiques à l'œuvre dans le patronage, même si les distinctions public / privé ; maison / Etat sont largement inopérantes à l'époque moderne. Le chapitre 4 est consacré aux sites institutionnels du patronage étatique. Le pouvoir royal, en France comme en Angleterre, nourrit un intérêt réel pour le monde mécanique. En témoignent notamment les sites d'expérimentations des Minories et de Vauxhall à Londres, où œuvrent plusieurs ingénieurs au service de la Couronne. Il n'y a pas, pour autant, d'administration des sciences et des techniques. Si on observe bien des charges spécialisées relevant de l'administration de l'Artillerie, de la Monnaie ou des Mines, tout indique que le patronage des hommes de science s'exerce depuis des charges sans dimension technique mais aux prérogatives larges, comme 
celle de chancelier. C'est en sa qualité de maitre des requêtes que l'Anglais Thomas Aylesbury, réputé patron des sciences, a pu appuyer les demandes de l'ingénieur Thomas Rudd ou du monnayeur français Nicolas Briot. Les monarchies entretiennent également une série d'emplois techniques : ingénieurs, enseignants ou artisans spécialisés. En France comme en Angleterre, si le mérite au combat et la compétence sont des critères décisifs de l'obtention d'une charge d'ingénieur, les jeux de la faveur restent prépondérants. La carrière du mathématicien anglais Richard Delamain illustre l'articulation habile de différentes activités : concepteur d'instruments mathématiques puis tuteur en mathématiques à la cour, ses positions lui permettant d'accéder grâce aux médiations de ses patrons au poste d'ingénieur sans avoir jamais livré bataille. Techniciens et savants sont mobilisés par l'Etat à deux fins, que Stephen Pumfrey et Frances Dawbarn ont résumées par les expressions "patronage spectaculaire » et «patronage utilitaire »3. Le premier de ces patronages est illustré dans cette enquête par le patronage hydraulique des monarchies anglaise et française, lesquelles, pour émuler l'art italien des jardins ou pour répondre à la demande édilitaire, encouragent les recherches des ingénieurs fontainiers. Le second type de patronage est illustré par la recherche sur les armes à feu, notamment sur les armes à répétition. Armuriers et arquebusiers œuvrent là à la frontière du divertissant et de l'utile, si bien que la distinction spectaculaire / utilitaire paraît quelque peu factice. Ces exemples montrent qu'une culture technique se consolide au sommet et dans les rouages de l'Etat dans les premières décennies du XVII' siècle.

Le chapitre 4 adopte une autre échelle d'observation et se propose de suivre l'origine, le déroulement et les conséquences d'une vive querelle scientifique du XVII ${ }^{\text {e }}$ siècle, la querelle des longitudes. Dès le XVI ${ }^{\text {e }}$ siècle, à l'instigation de la monarchie espagnole puis des Provinces-Unies, des récompenses avaient été proposées aux inventeurs d'une méthode fiable pour déterminer les longitudes en mer, que ces méthodes reposent sur des calculs astronomiques ou sur une horloge. La question s'apparente vite à un défi attirant farfelus et quadrateurs de cercle. Le chapitre restitue d'abord quelques unes des propositions adressées à la monarchie française au début du XVIIe siècle. Dans les années 1630, le contexte politique apparaît favorable aux donneurs d'avis : le principal ministre Richelieu s'est en effet octroyé la charge de surintendant de la navigation et réunit une commission scientifique pour établir le méridien de référence. C'est alors que le médecin et astrologue Jean-Baptiste Morin, professeur au collège royal, affirme lors d'une conférence du Bureau d'Adresse de Renaudot avoir découvert le secret des longitudes. Il obtient de voir sa découverte jugée par un comité d'experts ad hoc nommé par Richelieu, démonstration dont il espère " condigne récompense ». A l'issue de deux examens, la proposition de Morin est sévèrement critiquée, étant estimée à la fois impraticable et peu novatrice. Le chapitre ne s'intéresse pas tant au contenu de la controverse scientifique, laquelle s'épuise rapidement, qu'à ses acteurs, à leurs motivations et à leurs stratégies. La querelle et son écho dans la bonne société du temps ont été saisis comme un «moment effervescent» par les différents protagonistes pour reconfigurer les hiérarchies internes au monde savant. C'est ainsi que Morin s'attire l'hostilité d'un mathématicien écossais, James Hume, qui cherche à capitaliser sur la notoriété de la querelle pour obtenir une place à la cour. La dispute acerbe qui s'ensuit délaisse bientôt tout prétexte scientifique pour se concentrer sur les questions d'honneur et de statut. Morin, pour contrer l'expertise de ses juges, en appelle à d'autres formes d'accréditation : témoignages de personnes de qualité, « soutiens » d'autorités du temps comme Galilée ou Gassendi, dont les propos sont en fait tronqués et réagencés pour mieux satisfaire aux objectifs de Morin. Plusieurs indices montrent que ce sont les ressources du patronage que se disputent 
les protagonistes de la querelle, et notamment le patronage de Richelieu. Le chapitre revient également sur le rapport ambigu de Morin aux jeux du patronage. Né dans une famille de petite noblesse de cloche, il tente de se faire une place dans le monde en passant d'un protecteur à l'autre. Dans une publication tardive, il affirme avoir eu 16 maîtres qu'il quitta pour la plupart en mauvais termes. La querelle des longitudes, parce qu'elle met en scène les âpres rivalités interpersonnelles au sein d'une société de cour, présente donc l'aspect d'un drame du patronage.

Une troisième partie revient sur une autre forme de protection, le privilège d'invention. Le dossier documentaire entourant ces privilèges d'invention s'est étoffé presque naturellement au cours de l'enquête doctorale tant les points d'intersection avec les autres formes du patronage sont nombreux. Parmi les ingénieurs, techniciens ou mathématiciens qui peuplent les premiers chapitres, nombreux sont ceux qui ont sollicité pour des soi-disant inventions un privilège royal, c'est-àdire un monopole commercial assorti d'une protection contre les contrefacteurs. Autour de ces privilèges, se constituent des entreprises que nous avons qualifiées de techniques, car elles prétendaient à l'exploitation commerciale d'un procédé nouveau.

Le chapitre 5 revient sur les circuits d'obtention des privilèges. Le point de départ en est la rédaction d'un placet, texte convenu qui s'apparente à une écriture administrative davantage que courtisane. Ce n'est pas tant le contenu du placet qui importe que la main qui va la présenter au monarque. L'octroi du privilège est en effet une grâce du souverain, mais les premiers interlocuteurs des suppliants sont les secrétaires d'Etat en France et les maîtres des requêtes en Angleterre. La confiance d'un officier permet sans nul doute d'accélérer la procédure et de lui assurer une issue heureuse. Des commissions spécialisées existent bel et bien, même si l'historiographie française a surévalué leur importance, y voyant une anticipation de la procédure d'examen préalable que met en place l'Académie des Sciences à la fin du XVII ${ }^{\mathrm{e}}$ siècle. Ces commissions se prononcent rarement sur la faisabilité d'une affaire, concentrant leur propos sur son opportunité juridique ou sur ses dangers éventuels. Le principal souci des juristes ou des représentants des corps de métiers qui constituent les experts est de ne pas léser les droits existants. En l'absence d'arbitrage technique approfondi, ce sont donc les jeux de la faveur qui font la décision.

Ce caractère permet de rendre compte de la structure des entreprises privilégiées, objet du chapitre 6. Les entrepreneurs sont d'abord des courtisans ou des individus disposant de connexions auprès des preneurs de décision. En France, les commensaux et les officiers des Maisons royales sont surreprésentés parmi les récipiendaires des privilèges. En Angleterre, c'est le cas des serviteurs de la Chambre à coucher (département de la Maison du roi) et de l'entourage écossais du souverain, Jacques $\mathrm{I}^{\mathrm{er}}$ favorisant franchement ses compatriotes. L'entreprise privilégiée est cependant marquée par une grande diversité. Il y a peu en commun entre les affaires individuelles un peu hasardeuses d'un Etienne de Villebressieu, ingénieur ami de Descartes aux multiples initiatives, et les investissements multiples et croisés qu'on peut observer en Angleterre autour d'un groupe d'Allemands et d'Hollandais (Prusen, Rotsipen, Drebbel), liés aux munitionnaires ou au drainage des marais des Fens. Le chapitre aborde deux cas d'entrepreneurs exceptionnels de part et d'autre de la Manche. En Angleterre, le valet de la Chambre à coucher et horloger du roi David Ramsey se distingue en recevant près d'une dizaine de privilèges pour des affaires de métallurgie ou de pompes et reçoit au même moment d'importances concessions minières dans tout le royaume. Cet alchimiste, typique de la figure de l'homme à projet, peut être rapproché de Pierre de Beringhen, qui œuvre en France un peu plus tôt dans le siècle. Armurier s'étant attaché au service d'Henri IV en qualité de premier valet, Beringhen multiplie 
également les investissements, dans les mines, les mûriers, les assèchements de marais, les sociétés de tourbage ou la métallurgie du cuivre. Son action économique multiforme n'est en définitive qu'un produit du patronage du premier Bourbon.

Un dernier chapitre est consacré aux heurts et malheurs de l'entreprise privilégiée. Trois études de cas montrent tout le poids de la faveur dans le destin des projets entrepreneuriaux et restituent le climat affairiste qui entoure les privilèges d’invention. A Londres, plusieurs entrepreneurs avaient proposé au début du XVIIe siècle leurs services pour draguer le fonds de la Tamise, souvent en réemployant le sable et la vase pour fournir du ballast aux navires. Mais l'entrepreneur qui s'impose dans ce secteur concurrentiel, Thomas Smyth, ne parvient à le faire que parce qu'il a l'oreille du secrétaire d'Etat John Coke. Ce dernier lui procure le privilège, en tire une proclamation royale plus solennelle encore et fait obstacle aux rivaux qui bientôt menacent Smyth. Devant les difficultés juridiques et logistiques, l'entrepreneur est finalement lâché par son patron et l'entreprise périclite. Le deuxième cas abordé - les machines à remonter les bateaux - a déjà fait l'objet d'un article auquel nous nous permettons de renvoyer : il montre une noblesse d'affaire à l'action, n'hésitant pas à investir son crédit et ses deniers dans des machines de halage au fonctionnement hasardeux. Le patronage revêt ici la forme de ce que nous qualifierions aujourd'hui de conflit d'intérêt: le secrétaire d'Etat Henri de Guénégaud délivre un privilège à un entrepreneur au début des années 1660 tout en y investissant, par actes notariés, plusieurs de milliers de livres tournois 4 . Enfin, le dernier exemple développé est celui des sociétés de tourbage, dont on suit le cheminement ardu depuis une première tentative dans les années 1610. Le principal protagoniste de l'affaire est ici un avocat réformé, Charles de Lamberville, qui mobilise avec succès plusieurs instances d'expertise et d'accréditation (notamment la Gazette Renaudot) pour mettre en œuvre un projet de réforme économique du royaume de plus en plus ambitieux. Ces trois cas montrent que la maîtrise des forces sociales et institutionnelles est indispensable à l'émergence des entreprises privilégiées : la volonté de conquérir un marché se nourrit de la faveur bien plus que de l'innovation. Mais c'est là toute la fragilité de ces initiatives préindustrielles: leurs promoteurs pèchent par inexpérience ou incompétence techniques et la culture entrepreneuriale n'est encore qu'en gestation.

Ce travail aboutit à plusieurs conclusions. La thèse souligne d'abord que les formes et les foyers du patronage sont bien plus divers que l'historiographie focalisée sur les grandes maisons aristocratiques peut le laisser supposer. Ces modalités sont autant d'opportunités pour des acteurs aux profils eux-mêmes très variés. L'enquête met en valeur un monde peu connu de l'historiographie française, fait d'ingénieurs, d'enseignants, d'entrepreneurs, de techniciens et montre ses connexions, parfois souterraines, avec le monde plus érudit de la « république des sciences ». Ce souci de construire le problème du patronage par le bas amène à reconsidérer l'institutionnalisation du monde savant: la création des Académies, en France comme en Angleterre n'est pas seulement une monopolisation du mécénat par les couronnes, mais aussi une réponse par un État technoscientifique émergent aux sollicitations de ses sujets.

1 FRÄNGSMYR Tore (éd.), Solomon's House Revisited. The Organization and Institutionalization of Science, Canton (Mass.), Science History Publications, 1990.

2 MERSENNE Marin, Correspondance du P. Marin Mersenne, religieux minime, vol. XVI, Paris, CNRS, 1987, p. 57, Jacques Le Tenneur à Marin Mersenne, 16 janvier 1648.

3 PUMFREY Stephen, DAWBARN Frances. "Science and Patronage in England, 1570-1625 : A Preliminary Study », History of Science, Volume 42, 2004, p. 139-143.

4 RUELLET Aurélien, "Les 'machines à remonter les bateaux' en France au XVIIe siècle », Artefact. Techniques, histoire et sciences humaines, $\mathrm{n}^{\circ} 1$, 2013, p. 201-215. 